\title{
Current trends in splinting the hand in children with neurological impairments
}

\author{
Lauren Michelle Hepworth, B OT (UKZN); MHandRehabilitation (UKZN) \\ Lecturer Discipline of Occupational Therapy, School of Health Sciences, University of Kwazulu-Natal (Westville Campus)
}

Pragashnie Govender, B OT (UKZN), M OT (UKZN); PhD (UKZN)

Lecturer Discipline of Occupational Therapy, School of Health Sciences, University of Kwazulu-Natal (Westville Campus).

Gina Rencken, B OT (UP) M OT (UFS)

Lecturer Discipline of Occupational Therapy, School of Health Sciences, University of Kwazulu-Natal (Westville Campus)

Aim: The study aimed to explore Occupational therapy clinicians' current splinting practises in hand function intervention for children with neurological impairments within the South African context.

Methodology: A quantitative cross-sectional design with use of an electronic questionnaire served to address the objectives of this study. The sample included occupational therapists working within the paediatric neurology field in South Africa. The study sample was accessed via convenience and snowball sampling in order to target therapists specifically working in the area of paediatric neurology.

Results and Discussion: Forty therapists from around South Africa (majority from KwaZulu Natal) completed the survey in its entirety. Therapists splint for various reasons and splints are considered effective in neurological cases. The three most prevalent splints were the functional resting, thumb abductor and anti-spasticity splint with the purpose of splinting mainly reported to maintain or improve range of motion (ROM). In this study, therapists appeared to consider knowledge, competency and experience prior to the availability of resources when rationalising the choice to splint.

Conclusions: This study provides insight into the splinting practices amongst occupational therapists who work with neurologically impaired children. Despite the controversy that surrounds splinting in neurology, therapists are using splints in their management with a number of factors considered during the decision-making process.

Key words: Splinting, paediatrics, neurology, occupational therapy

\section{INTRODUCTION}

Splinting for children or adults with a neurological impairment has often been considered a controversial area of practice amongst Occupational therapists utilising a neurophysiological approach ${ }^{1,2}$. Authors in the field of neurological rehabilitation have recommended that therapists base practice on scientific evidence ${ }^{3,4}$. In the past, therapists have been noted to focus more on compensating for impairment in clients with neurological impairment, but due to the increasing knowledge of neuroplasticity; therapists are encouraged to focus rather on minimising impairment and increasing function through the training or retraining of activities of daily living $(A D L)^{3}$. 'Neurological impairments is a term used to describe conditions where there has been an insult to the central nervous system (CNS). The CNS mainly develops during pregnancy and in the first five post-natal months. It is during this period when the 'immature brain' is most vulnerable to malformation, infection, damage or disruption, thereby leading to disorganisation of the $\mathrm{CNS}^{5}$. These insults to the brain may result in conditions such as cerebral palsy, pervasive developmental disorders, genetic disorders, degenerative conditions, and may also include traumatic brain injuries and HIV related conditions ${ }^{6}$. Due to the minimal scientific literature on the clinical benefits and efficacy of splinting in neurological conditions for both adults ${ }^{7}$ and children ${ }^{8}$, therapists commonly use their experience to justify their reasoning for splint application when working with the neurologically impaired population?. This study thus explored the current splinting practices of Occupational therapists within the South African context with children with neurological impairments.

\section{LITERATURE REVIEW}

Splinting in neuro-rehabilitation is typically justified through what therapists have found to work best in order to: (i) normalise tone in order to prevent or correct contractures, (ii) increase function within the affected arm through improving movement whilst protecting the integrity of the joint and (iii) decrease pain ${ }^{10}$. Although there is little research evidence to suggest that splinting in neurorehabilitation achieves these aims, the majority of therapists continue to recommend splints based on their clinical experience and judgement as opposed to the research evidence ${ }^{11}$. Two previous studies focussed on splinting trends for clients with stroke. These studies indicate that the main aims of splinting are to prevent soft tissue contractures ${ }^{12}$ as well as improve hygiene and functional needs, maintain muscle length, and prevent deformity and contractures ${ }^{13}$.

Occupational therapy is often associated with functional aims ${ }^{13}$. A study done on splinting in neurology in South Africa however, indicated that therapists only suggested splinting if active movement is present in order to promote function ${ }^{14}$. Additionally therapists use splints in order to decrease pain and to maintain the hand or arm in a comfortable position"'. This was, however refuted by Lannin et al ${ }^{15}$, who found that there was no significant impact on the decrease of pain with the use of splints. A study done with eight stroke participants investigated the effects of different splinting regimes on spasticity reflected no significant difference in tone reduction, but rather noted that splints could increase the tone, being largely influenced by the duration of wear ${ }^{2}$.

Various studies have begun to identify the value of a number of splints in neurological conditions ${ }^{1,2,16,17}$; however, there is currently no available literature that documents the splints used in practice 
within a South African context in the paediatric neurologically impaired population. A functional resting splint was indicated for hands that have moderate to severe increased tone and severely decreased tone, for the prevention of contractures and for hygienic purposes ${ }^{16}$. The functional resting splint is usually positioned in $30^{\circ}$ extension at the wrist, $50^{\circ}$ flexion in the metacarpophalangeal joints and progressive $10^{\circ}-30^{\circ}$ degree flexion at the interphalangeal joints ${ }^{18}$. The effectiveness of the functional resting splint in a stroke population was examined by Pizzi et al. ${ }^{17}$. Although there was no control group, the authors found that the use of functional resting splints on forty subjects with a stroke were beneficial, measured by an assessment of spasticity, passive range of motion (PROM), pain, presence of spasms and a subjective report of comfort. There was also a significant difference in passive wrist extension and spasms were found to have decreased ${ }^{17}$. The wrist extension splint has also been used in patients with neurological impairments. It has however been indicated in a study ${ }^{19}$, that patients wearing a neutral wrist splint were more compliant than those wearing a wrist extension splint. No significant impact on function was noted ${ }^{19}$. Other prominent splints that have been indicated in the scientific literature include the weight-bearing splint, the anti-spasticity splint and the serpentine splint. The weight-bearing splint positions the wrist in approximately $90^{\circ}$ extension with appropriate digit extension but is usually used during tasks requiring weight-bearing ${ }^{16}$. The anti-spasticity splint, found to be most effective in a South African study ${ }^{14}$, allows for the extension of the wrist with finger and thumb abduction achieved by finger troughs 9 . The properties thought to contribute to its efficacy included placing the hand in a functional position, improved hygiene and providing prolonged stretch. The serpentine splint ${ }^{16}$ wraps around the thumb from just below the distal palmar crease on the hypothenar side, across the dorsum below the metacarpophalangeal joints in order to wrap around the thenar eminence through the web space. It is designed to minimise adduction contractures of the thumb, but as there is wrist movement, it serves to assist in stabilisation of the thumb during activities $^{20}$. A randomised-controlled trial used three splinting conditions, namely no splint, a dynamic and a static splint to investigate the effects of splints in children with increased tone. The sample included ten children with spastic hemiplegia, and a control group $(n=10)$ that used both their dominant and non-dominant hands. The study revealed that a dynamic splint when prescribed for children with neurological impairments, showed a better post-splint grip and fine motor skills as opposed to a static splint ${ }^{21}$.

Splints are typically constructed from thermoplastic material. In recent times, the use of neoprene or Lycra has been used in the construction of what is known as "soft splints". A soft splint is considered to be advantageous as it provides prolonged stretch to the muscles to normalise increased tone, whilst not limiting movement ${ }^{22}$. Although studies have begun to document the value of splinting in neurological conditions as mentioned above, these remain limited with insufficient scientific evidence on their efficacy. Moreover, the frequency and type of splinting used in paediatric conditions remain unknown in the South African context.

\section{METHOD}

This study aimed to explore the current splinting practises of Occupational therapists in hand therapy intervention for children with neurological impairments within the South African context. A cross sectional survey was conducted nationally amongst Occupational therapists registered with the Health Professional Council of South Africa (HPCSA).

\section{Population and Sampling}

The target population ( $\mathrm{N}$ ) for this study included all Occupational therapists working in the field of paediatric neurological rehabilitation in South Africa. There was, however, no prior knowledge of the number of therapists $(N=7234)^{23}$ that worked specifically with neurologically impaired children. The institute of private Occupational therapy practitioners (INSTOPP) assisted in providing statistics on the number of private therapists working with neurologically impaired children, as practitioners belonging to this organisation are able to self-report and indicate their field of practice in the organisation's database. There is however, no specialist register for Occupational therapists with the HPCSA and therefore no record or indication of the number of public service employees who worked in the field exists. Although a number of hand therapists could be accessed on the Internet via the South African Society of Hand Therapists (SASHT), this was not accurate as not all therapists would work with children and not all hand therapists are necessarily registered on SASHT. INSTOPP and SASHT are voluntary member organisations, and annual membership fees apply. Due to these reasons, the target population could not be ascertained, affecting the calculation of the sample size. As this study targeted specifically those Occupational therapists who splint the paediatric neurologically impaired hand, sampling procedures were amended, as there were no available records of non-private practitioners working in specific fields. Convenience and snowball sampling ${ }^{24}$ was thus employed, to reach as large a proportion of the target population as possible and was based on the following selection criteria: Therapists who: (i) were registered with the HPCSA as community service or independent practitioners, (ii) had undergraduate and/ or postgraduate degrees in Occupational therapy, and (iii) were currently working with children with neurological impairments at least twice per week.

Due to the self-administered nature of the survey, there was a potential for a non-response bias ${ }^{24}$. After four months, a total of 72 responses were collected, of which 32 were partially completed and 40 were completed in full.

\section{Instrument Development}

Themes from questionnaires designed in previous studies in Can$\mathrm{ada}^{25}$, Ireland ${ }^{11}$ and the United Kingdom ${ }^{12}$ were considered. These included the frequency of splint use for children with spasticity and the reasons for the certain types of splints and treatment goals associated with splinting. This information was expanded to include questions that considered factors that influence decision-making for splinting children with neurological impairments as well as an exploration of what therapists viewed as their needs in this area in terms of further information or practical experience. Themes and questions were adapted and assisted in shaping the layout and content of the study.

The final survey was structured as follows:

* Biographical Data (Section A) including postgraduate training, years of experience and the sector that therapists were employed within.

* Background on splinting (Section B) in terms of experience in neurological rehabilitation and work load

* Types of splints prescribed (Section C). This examined the materials used, what influenced their choice of material as well as types of splints prescribed.

* Client factors that influence decision-making (Section D) involved the most common neurological diagnostic groups for which splints were prescribed as well as the rationale for splinting.

* Personal factors that influence decision-making (Section E). This section explored the factors that therapists take into consideration such as their confidence, skill and knowledge when splinting for neurologically impaired children.

\section{Pilot Study}

A pilot study on ten Occupational therapists was conducted in order to refine the survey. The results of the pilot study were analysed and amendments effected prior to the survey link being emailed to the target population ${ }^{26}$.

\section{Validity Testing}

Face validity was ascertained using individuals not trained within the Occupational therapy profession. They were provided with 
an opportunity to review the survey prior to the expert review in order to assess for syntax and grammatical errors as well as to check the clarity and understanding of questions ${ }^{27}$. Content validity was ensured through both the expert review and the pilot study. In order to ensure reliability, it was important to ensure that there was no ambiguity so that each question could be answered in the same manner ${ }^{28}$.

Contact details of Occupational therapists across South Africa were collected from Occupational therapy affiliated organisations (SSASHT, INSTOPP and SANDTA) or forums as well as through contact with Occupational therapy departments at South African Universities. A statistician was consulted to ensure accurate data analysis. The survey link was then distributed via email. The therapists were asked to complete the informed consent if they chose to participate prior to the survey. Reminders were sent out on a fortnightly basis and the survey was open for a period of six months.

\section{Data analysis}

The summary data - collected from the survey - were exported from the SurveyMonkey site onto an Excel(C spreadsheet using words. In order to allow for easier analysis, the data were changed to numeric values where appropriate. A statistician was consulted for data analysis with the principal author being responsible for the final analysis. Data were arranged into nominal (for example age, years of experience, work sector) and ordinal data (Likert scales). The main findings were organized and represented as either bar graphs, or pie charts. The Likert scale data were analyzed for central tendency and summarized by means of a mode ${ }^{29}$.

\section{Ethical Considerations}

Ethical clearance was first obtained through a Biomedical Research Ethics Committee (BREC) at the University of Kwa-Zulu Natal (Ref: BE3 I $9 / 14$ ). The ethical principles of respect for persons (informed consent, issues around beneficence and confidentiality) and justice (voluntary participation, right to withdraw, scientific honesty and integrity) were upheld in this study.

\section{RESULTS}

\section{Demographics of participants}

The majority of therapists were within the 26 to 30 year age range (33.8\%). The responding therapists were from a variety of working sectors with over half $(52.3 \%)$ reporting employment within an acute public hospital setting. This was followed by $29.2 \%$ working in private practice and the remaining $18.4 \%$ being minimally represented in other working sectors. The majority of the respondents were based in Kwa-Zulu Natal (36.9\%) and the Western Cape (26.2\%), followed by $16.9 \%$ based in Gauteng. There was only a small representation of therapists from the North West Province (I.5\%), Northern Cape (I.5\%) and Limpopo (I.5\%). Work experience spanned from one to more than 20 years, with the majority of the therapists (45.5\%) only having one to two years' experience. The majority had only been working with children with neurological impairments for a period of one to two years $(38.9 \%)$ and $24.1 \%$ reported more than 10 years' experience. (See Figure I below)

\section{Background on splinting experience}

The exploration of reasons for not splinting were considered an important part of the study, as it provided more information on the possible contributing factors to decision making and clinical use of splints in children with neurological impairments. The following influential factors were explored: departmental policy, evidence-base of splints, confidence, preference for other techniques, availability of materials and supervision. The main factors that had little effect on the therapists' decision to splint a child with a neurological impairment included the presence or absence of any departmental policy $(84.6 \%)$. This was followed by a lack of supervision $(64.8 \%)$ with only $11.8 \%$ indicating that this was often a problem when deciding whether or not to splint. Most therapists appeared to seldom consider these factors, suggesting that they would choose to splint based on their own clinical decision-making. They did show a preference however, for other techniques, such as the use of neurodevelopmental techniques (NDT), with $33.3 \%$ stating this often

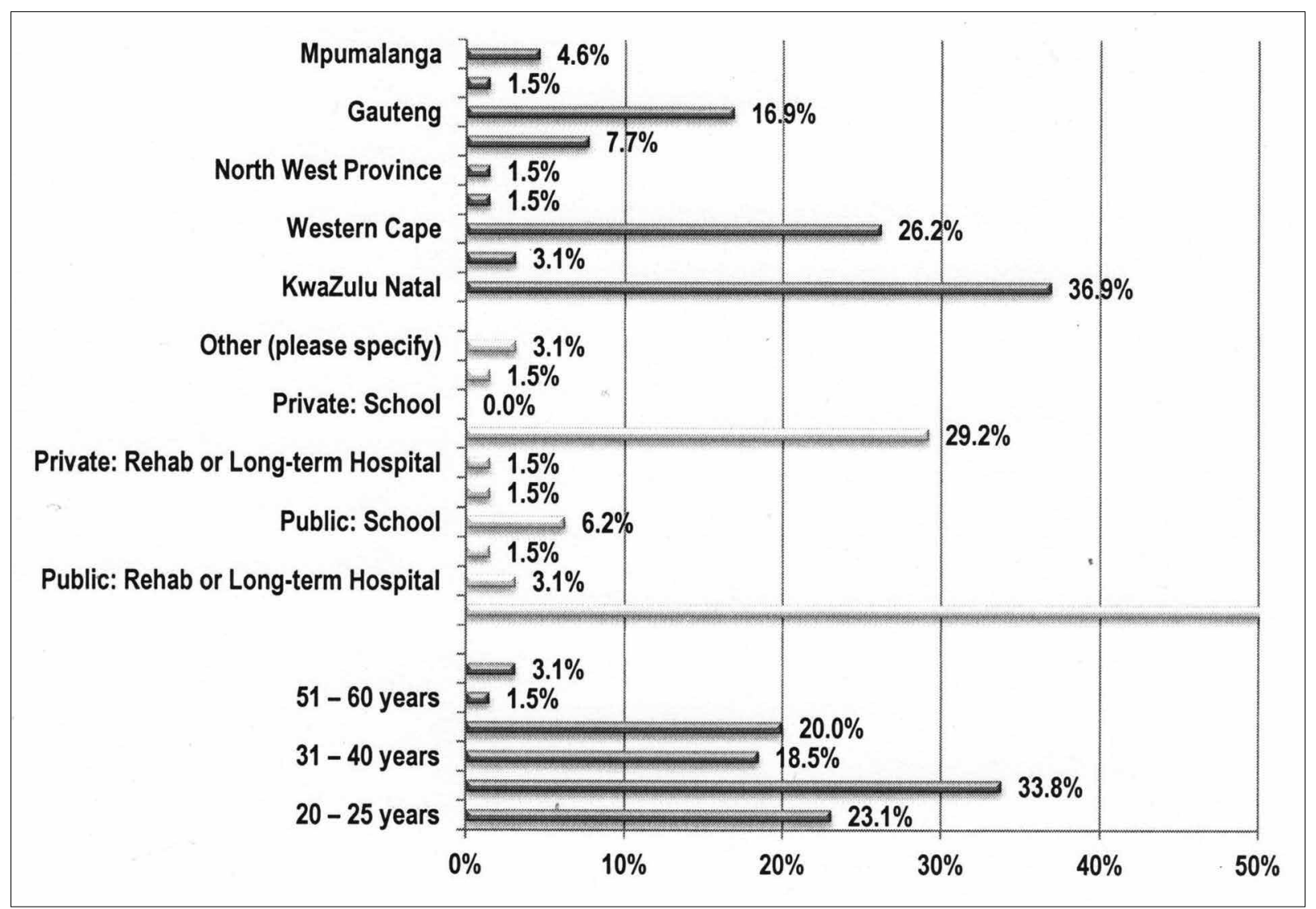

Figure I: Demographic information of Survey Participants $(n=65)$ 
influenced their decision-making in terms of splinting, and $38.8 \%$ stating that it sometimes influenced their decision. The therapists showed little consideration for evidence-based research, with only $18 \%$ indicating this as an important factor. Decreased confidence and a lack of materials did not influence the decision to splint as only $1 \mathrm{I} .8 \%$ considered both these factors.

Therapists stated that they learnt how to splint from a variety of sources, with the majority doing so in their own time either through textbooks (6I.5\%) or through self-teaching (59.6\%). Only $50 \%$ stated that they had learnt to make splints for children with neurological impairments during their undergraduate training. The least common resource was postgraduate training (I5.4\%).

\section{Preference of splinting material}

Most therapists' preferences for material were a combination of thermoplastic and neoprene $(57.5 \%)$, followed by a preference of neoprene $(27.4 \%)$ (Figure 2 ). This was influenced by the following factors: $65 \%$ felt that their choice of material led to better results, $60 \%$ chose material based on the ease of application with $47.5 \%$ feeling that it was important for better compliance. Only $7.5 \%$ based their choice of material on cosmetic appeal. All (I00\%) agreed that they preferred to custom-make their own splints as opposed to using off-the-shelf products.

In terms of the type of splints preferred for children with neurological impairments, therapists had the option of selecting multiple answers. Figure 3 below illustrates the two most popular splints, namely, the functional resting splint $(80 \%)$ and the neoprene thumb abduction splint $(80 \%)$. There were four responses to the 'other' category. These included a thumb opposition splint, wrist extension splints, a post-box splint and a neoprene splint similar to the soft abduction splint, but which includes the wrist.

\section{Rationale for splinting based on client's condition}

Reasons for splinting were explored using a five-point Likert scale. This scale was collapsed during the data analysis to a three-point scale for ease of interpretation. The categories 'never' and 'seldom' were reduced to 'seldom'; 'sometimes' remained the same and 'very often' and 'always' were reduced to 'often'. More than half of the therapists considered every factor listed when splinting their clients (Figure 4). Maintenance/improvement of ROM (97.5\%), prevention of contractures $(92.5 \%)$ and the compliance of the caregiver $(90 \%)$ reflected the highest frequencies when splinting a neurologically impaired child. (See Figure 4 on page 14)

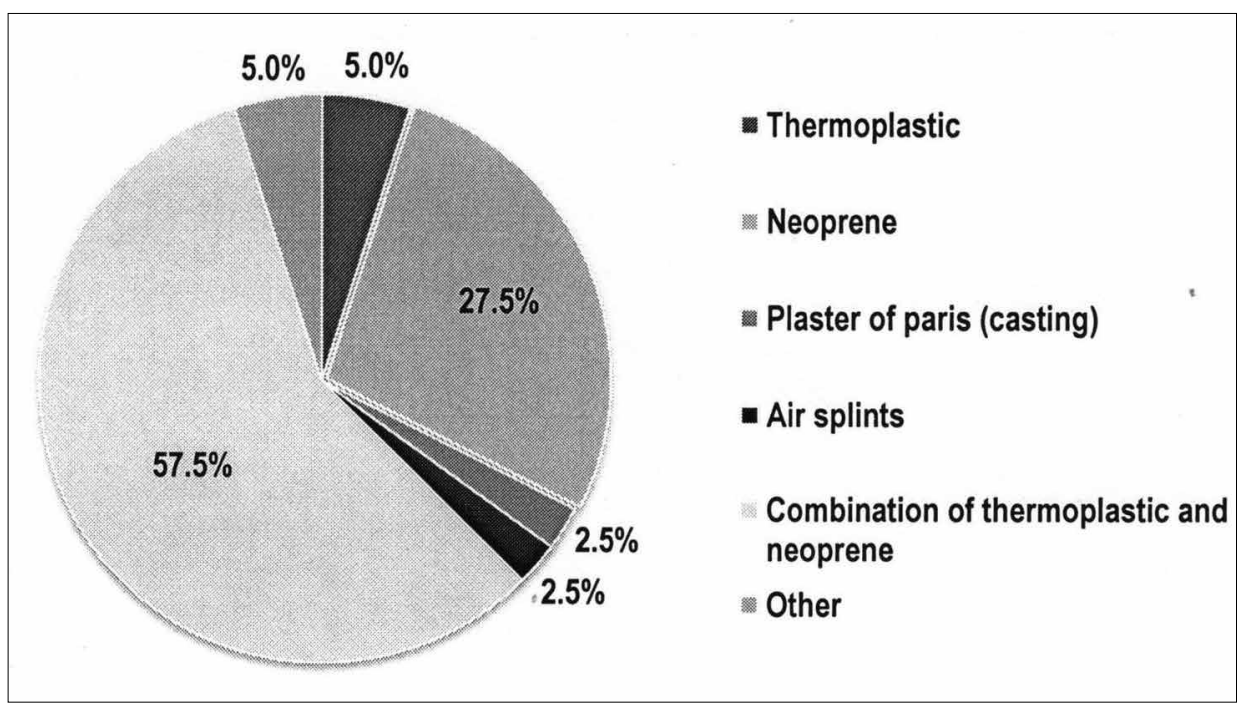

Figure 2: Preferred Material for Splinting the child with NI $(n=40)$

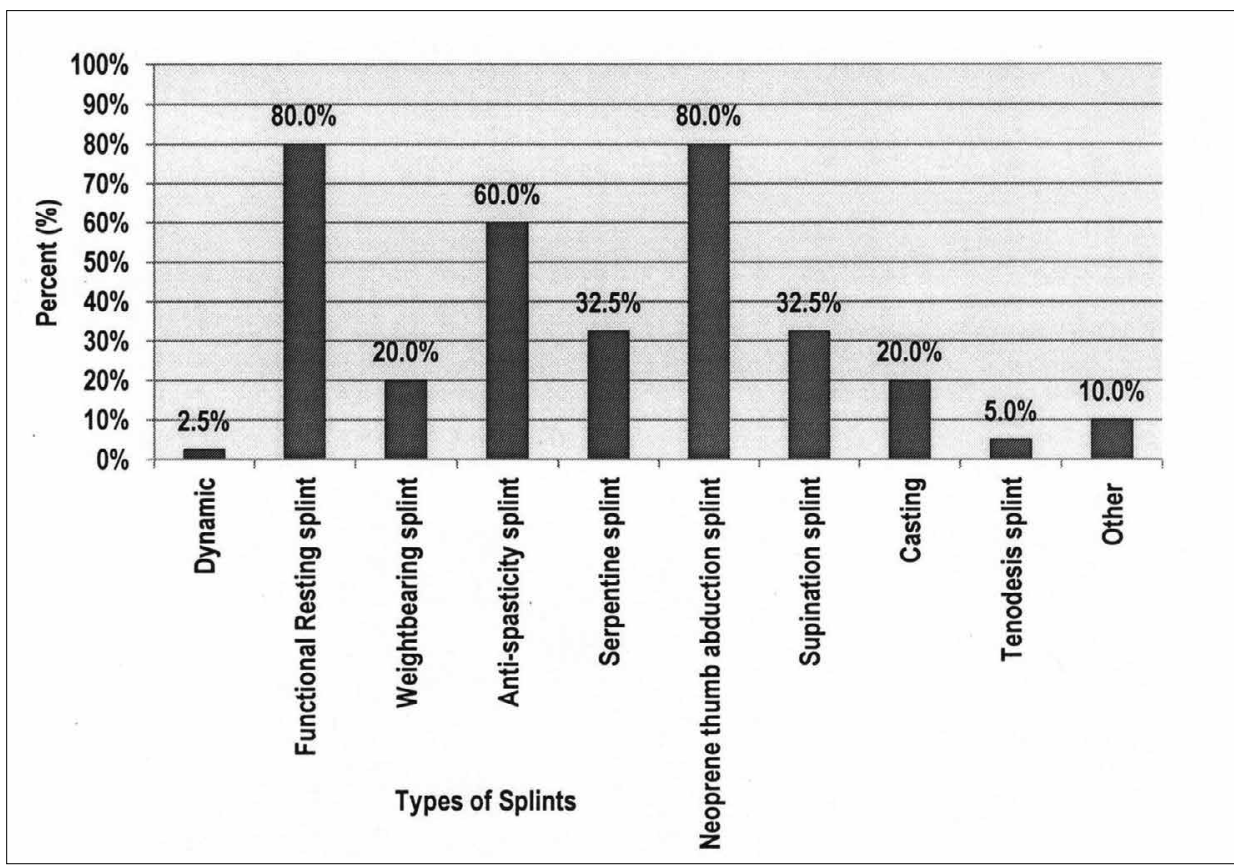

Figure 3: Preferred splints for the Child with NI $(n=40)$

\section{Rationale for splinting according to personal factors}

Personal factors considered during splinting included knowledge of the condition, therapists' experience with splinting, and their feelings of competency, resources available and time (Figure 5). A five-point Likert scale was again collapsed during the data analysis to a threepoint scale for ease of interpretation. It was found that the knowledge of the condition $(65 \%)$ was the main factor that was often considered during splinting. This was followed by almost half of the therapists considering that competency $(47.5 \%)$ and experience (45\%) should be taken into account when splinting. (See Figure 5 on page 14)

\section{DISCUSSION AND IMPLICATIONS}

This study describes the current practices of Occupational therapists in splinting of children with neurological impairments. All therapists in this study preferred the use of custom-made splints. This was different from other studies in which 30\% of Occupational therapists prefer off-theshelf static splints ${ }^{12}$ and another showed differences in preferences amongst different groups of Occupational therapists ${ }^{\prime \prime}$. Although prefabricated splints can be adjusted for each individual patient, it has been found that ill-fitting, off-theshelf splints can lead to an increase in psychosocial fears and an increase in tone ${ }^{7}$. A custom-made splint, however, is specific and individualised. Given the current resource-strained contexts that many South African Occupational therapists work in, the availability of custommade splints may be limited, and may thus affect therapists' affinity to custom-made splints.

Over half of the therapists stated that they would choose materials based on 


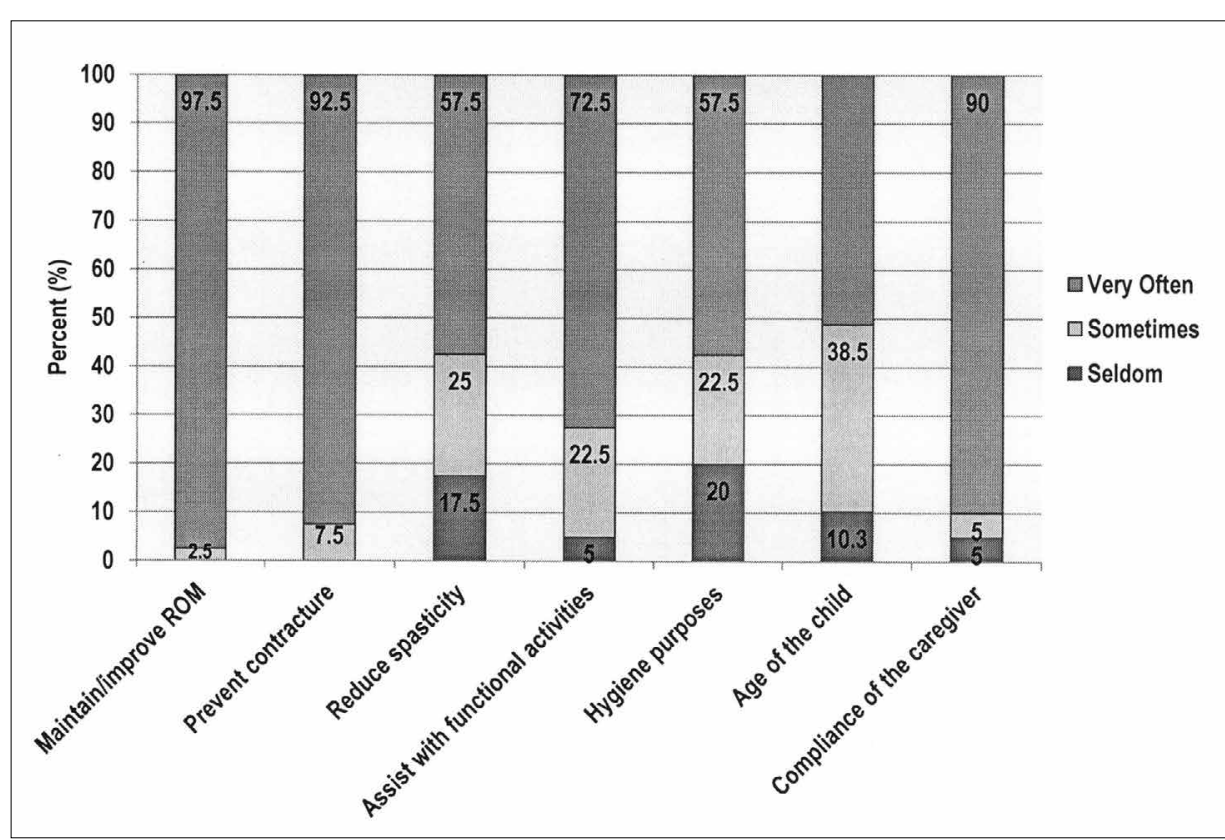

Figure 4: Client Factors when Splinting a Child with NI $(n=40)$

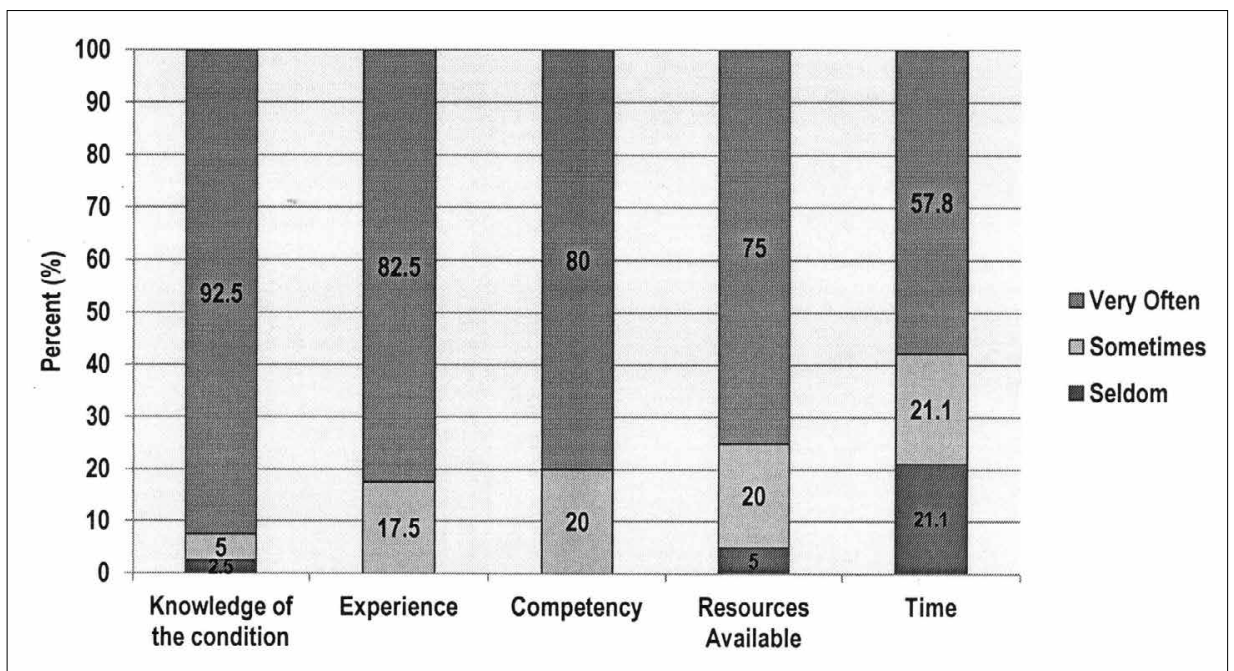

Figure 5: Personal Factors considered in Splinting a child with NI $(n=40)$ of evidenced-based research to support their stance. This once again shows a discrepancy between the evidence and therapists' perception of the benefits of splinting in children with neurological impairments.

Therapists showed a marked preference for three splints despite the lack of scientific evidence documenting the benefits of each splint (cf. Figure 3). The functional resting splint ${ }^{16}$ is the most preferred and second most prescribed splint for children with neurological impairments despite the lack of scientific evidence ${ }^{10}$.

The second most prescribed splint, but the most preferred splint is the neoprene thumb abduction splint. New studies are being done in order to assess the efficacy of this soft splint. Soft splints allow for movement ${ }^{22}$, as opposed to the functional resting splint, whilst still providing a prolonged stretch. In terms of normal development, active movement is encouraged from an early stage. Grasps begin from the ulnar side of the hand and thereafter move to the radial side of the hand $^{31}$. Thus the use of a neoprene-based splint may address this as the design allows for more normal movement and exploration, which is essential for a child's overall development.

The other prominent splint that is prescribed in neurology is the anti-spasticity splint $^{25}$. Although usually relating to adult neurology - as it was found that therapists viewed this splint to be most effective post-stroke - it has also been used to allow for improved hygiene ${ }^{14}$, which is an important aim for children with forming contractures.

Only a small percentage of therapists $(2.5 \%)$ choose to prescribe dynamic splints despite dynamic splinting having good results in assisting in active movement $^{32}$. This is in keeping with a study in which the researchers postulate the under-utilisation of dynamic splinting ". In a randomised-controlled trial, it was found that both grip and fine motor skills improved after dynamic splinting as compared to static splinting ${ }^{2 !}$.

Occupational therapy is often associated with functional aims, and therefore the main goals of splinting should reflect a decisionmaking process in view of Occupational therapy practices. A number of client-factors affected the therapists' decision to use a splint in order to best enhance function. Knowledge about different neurological conditions was found to be important as this could guide future splinting protocols or guidelines ${ }^{12}$. The current research survey found that therapists, whether using thermoplastic or soft splints, mainly splinted children with cerebral palsy $(72.5 \%$ and $80 \%$ respectively) as well as traumatic brain injury, where $62.5 \%$ used thermoplastic splints and $42.5 \%$ used soft splints. It is interesting to note that therapists made use of splints in pervasive developmental disorders and future studies should investigate this further.

The three most common considerations when splinting as reported by the therapists in terms of client factors included maintenance/improvement of range of motion (ROM), prevention of contractures and compliance of the caregiver. This reasoning appears to have some universality as the main reasons stated for splinting was decreasing tone in the hand, preventing contractures and improving ROM. A similar finding was found in the survey done 
In the United Kingdom, which described that therapists mainly used splinting to prevent contractures ${ }^{13}$.

In order to achieve aims or goals in therapy, an Occupational therapist requires integration of theoretical knowledge and practice. Thus, Occupational therapists need knowledge of both the condition and types of splints available prior to splinting. Practical experience will therefore lead to greater feelings of competency and confidence. These factors were explored as personal factors that affect decision-making (cf. Figure 5). Almost all the therapists felt that knowledge of the condition was important when splinting as different conditions may have different presentations, for example, children with cerebral palsy may present with spasticity or dystonia ${ }^{5}$. This links to a therapist's knowledge about hand development and splinting as it may influence the choice of splint, the choice of materials or whether splinting would be beneficial. Although the therapists had seemed confident in terms of their knowledge of the different types of splints, almost half responded that their knowledge on neurological splinting is fair.

Therapists rely on their experience and clinical observations when deciding whether a splint would be of benefit "'. This was highlighted by the majority of therapists in this study who viewed experience as an important factor in their decision-making process with respect to splinting. This decision-making process has been emphasised, as therapists are less likely to base their choice of intervention on evidence-based practice as opposed to prior training and experience ${ }^{33}$. A feeling of competency is often related to both experience and confidence ${ }^{34}$. Almost half of the therapists in this study indicated that competency should be considered when splinting. This may be explained by therapists attributing increased skill to experience ${ }^{14}$ thus leading to a greater feeling of competency. Therapists' use of splints did not depend on available resources. In this study, therapists appeared to consider knowledge, competency and experience prior to the availability of resources ${ }^{25}$. This may be due to the South African context, especially as the majority of the therapists work in public hospitals; certain materials may not always be easily available.

Despite the low response rate, this study yielded findings that describe and provide insight into the types of splints and factors that influenced therapists' decisions when splinting children with neurological impairments.

\section{CONCLUSION}

In this study, Occupational therapists preferences in splinting in the neurologically impaired child were explored. Splinting is occurring as part of an Occupational therapists management, despite a lack of evidence-based research that details the benefits of neurological splinting. However, this appears to be on an infrequent basis with many therapists only prescribing between one to five splints per month despite the majority of therapists seeing above four children with neurological impairments per month. This may be due to a smaller number of children with neurological impairments forming part of the therapists' workload or alternatively as indicated by the therapists, other techniques also take preference. Both thermoplastic and neoprene materials are being used individually and in combination with each other. All the therapists in this study preferred to tailor-make splints in keeping with a client-centred approach as opposed to purchasing prefabricated splints. Three main splints were identified in terms of prescription frequency and preference. Therapists appear to consider many factors before splinting, but it is also clear that each therapist makes use of personal experience and theoretical knowledge in order to determine a decision regarding splinting. Scientific proof for the benefits of splinting in the child with neurological impairments is yet to be established, although therapists remain active in prescribing splints for the maintenance of range of motion, prevention of contractures and reduction of spasticity.

This study has provided data towards understanding current practices in splinting of neurological conditions in the paediatric population by Occupational therapists in South Africa and has assisted in identifying gaps that require further research and inquiry especially in terms of the efficacy of splinting in these particular conditions.

\section{REFERENCES}

I. Neuhaus BE, Ascher ER, Coullon BA, Donohue MV, Einbond A, Glover JM, Goldberg SR, Takai VL. A survey of rationales for and against hand splinting in hemiplegia. American Journal of Occupational Therapy. 198I Feb I; 35(2): 83-90.

2. Langlois S, Pederson L, MacKinnon JR. The Effects of Splinting on the Spastic Hemiplegic is Hand: Report of a Feasibility Study. Canadian Journal of Occupational Therapy. 1991 Apr I; 58(I): 17-25.

3. Sandin KJ. New Approaches to Neurorehabilitation: The Increasing Evidence Base. Minnesota Medicine. 2012 Jan; 95(I): 46.

4. Girard A, Rochette A, Fillion B. Knowledge translation and improving practices in neurological rehabilitation: managers' viewpoint. Journal of evaluation in clinical practice. 2013 Feb I; I9(I): 60-7.

5. Fairhurst $C$. Cerebral palsy: the whys and hows. Archives of disease in childhood-Education \& practice edition. 2012 Aug I; 97(4): I22-3 I.

6. National Children's Bureau. Information about neurological disorders. Early Support. 20I2. < http://www.ncb.org.uk/media/875206/ earlysupportneurologicaldisorderspart I final.pdf > (I5 Dec 20I5).

7. Greg Pitts D, Peganoff O'Brien S. Splinting the hand to enhance motor control and brain plasticity. Topics in stroke rehabilitation. 2008 Sep; 15(5): 456-67.

8. Samson-Fang L, Darrah J, McLaughlin J, Logan L, Hoon A, Msall M, Wiart L, Vogtle PL, Blackmore M, Australia W. A systematic review of the effects of soft splinting on upper limb function in people with cerebral palsy. An AACPDM Evidence Report Initial Publication In Database; October 2006.

9. Lohman M, Aragon O. Antispasticity Splinting in Coppard B, Lohman $\mathrm{H}$, editors. Introduction to Splinting: A Clinical Reasoning and Problem-Centred Approach. St. Louis: Elsevier Mosby. 2008: 353-377.

10. Lannin NA, Ada L. Neurorehabilitation splinting: theory and principles of clinical use. NeuroRehabilitation. 20I I Jan I; 28(I): 2I-8.

I I. Adrienne C, Manigandan C. Inpatient occupational therapists handsplinting practice for clients with stroke: A cross-sectional survey from Ireland. Journal of neurosciences in rural practice. 20 I I Jul I; 2(2): I4I.

12. Hoffman K, Baird T, Tuckey J, Marston L, De Souza L. Contemporary splinting practice in the UK for adults with neurological dysfunction: A cross-sectional survey. International Journal of Therapy and Rehabilitation. 2013; 20(I I): 559-566.

13. Khatri R, Logan P, Kay AD, Lehner AN. A national survey to evaluate current splinting practice for stroke survivors. Paper presented to: $37^{\text {th }}$ Annual Conference and Exhibition of the College of Occupational Therapists. 2013, Glasgow, UK,

14. Chazen LA. Understanding the occupational therapists use of splinting the affected hand of adult patients with neurological injuries. PhD study. University of the Witwatersrand; 2014.

15. Lannin NA, Horsley SA, Herbert R, McCluskey A, Cusick A. Splinting the hand in the functional position after brain impairment: a randomized, controlled trial. Archives of physical medicine and rehabilitation. 2003 Feb 28; 84(2): 297-302.

16. Gabriel L. Pediatric Splinting in Coppard B, Lohman H, editors. Introduction to Splinting: A Clinical Reasoning and Problem-Solving Approach. St Louis: Elsevier Mosby. 2008: 353-384.

17. Pizzi A, Carlucci G, Falsini C, Verdesca S, Grippo A. Application of a volar static splint in poststroke spasticity of the upper limb. Archives of physical medicine and rehabilitation. 2005 Sep 30; 86(9): 1855-9.

18. Mediroyal. Preformed Functional Splint. Mediroyal Splinting. 2010. <http://www.mediroyal.se/en/product/preformed-functionalsplint $>$ (2I Oct 20I3).

19. Lannin NA, Cusick A, McCluskey A, Herbert RD. Effects of Splinting on Wrist Contracture After Stroke A Randomized Controlled Trial. Stroke. 2007 Jan I; 38( I): I I I-6.

20. Darcera VB. Paediatric Splinting: Assignment Handed into Occupational Therapy Course. Velez College; 2012.

2I. Burtner PA, Poole JL, Torres T, Medora AM, Abeyta R, Keene J, Qualls C. Effect of wrist hand splints on grip, pinch, manual dexterity, and muscle activation in children with spastic hemiplegia: a preliminary study. Journal of hand Therapy. 2008 Mar 3I; 2 I (I): 36-43.

22. Fedrizzi E, Pagliano E, Andreucci E. Hand function in children with 
hemiplegic cerebral palsy: prospective follow-up and functional outcome in adolescence. Developmental Medicine \& Child Neurology. 2003 Feb I; 45(2): 85-9I.

23. Health Professionals Council of South Africa. 2015. http://isystems. hpcsa.co.za/iregister/. (I 3 January 20I4).

24. Trochim, W. Research Methods Knowledge Base. 2006. http://www. socialresearchmethods.net/kb/index.php. (9 Jan 2015).

25. Reid DT. A survey of Canadian occupational therapists' use of hand splints for children with neuromuscular dysfunction. Canadian Journal of Occupational Therapy. 1992 Apr I ;59(I): 16-27.

26. Domholdt E. Rehabilitation Research: Principles and Applications. Missouri: Elsevier Saunders; 2005: 228-235.

27. Litwin MS. How to measure survey reliability and validity. Sage Publications; 1995 Aug 3.

28. Bruce N, Pope D, Stanistreet D. Quantitative methods for health research: a practical interactive guide to epidemiology and statistics. John Wiley \& Sons; 2008 Sep 9.

29. Mogey N. So you want to use a Likert scale. Learning technology dissemination initiative. 1999 March: 25.

30. Hughes AA. The effect of neoprene thumb abductor splints on upper limb function in children with cerebral palsy (Doctoral dissertation).

31. Kumar DA, Senapoti DA. Effect of soft splinting for upper extremity on manual ability in children with spastic cerebral palsy. The Indian Journal of Occupational Therapy. 2012 Jan I; 44(I): 30-3.

32. Ter Schegget M. Grip Development in the Growing Child in Hovius S, Foucher G, Raimondi PL, editors. The Pediatric Upper Limb. London: Martin Dunitz. 2002: 38-55.

33. James SF. Contractures in orthopaedic and neurological conditions: a review of causes and treatment. Disability and rehabilitation. 2001 Jan I; 23(I3): 549-58.

34. Sweetland J, Craik C. The use of evidence-based practice by occupational therapists who treat adult stroke patients. The British Journal of Occupational Therapy. 200 I May I;64(5):256-60.

35. Holland K, Middleton L, Uys L. The sources of professional confidence in occupational therapy students. South African Journal of Occupational Therapy. 2012; 42(3): 19-25. 